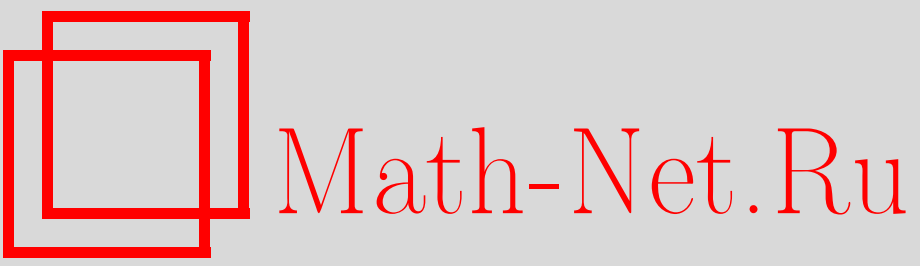

Г. М. Раимова, Вероятностное представление решения начально-краевой задачи для системы параболических уравнений, Теория вероятн. и ее примен., 2012, том 57, выпуск 4, 800-809

DOI: https://doi.org/10.4213/tvp4483

Использование Общероссийского математического портала Math-Net.Ru подразумевает, что вы прочитали и согласны с пользовательским соглашением

http: //www . mathnet.ru/rus/agreement

Параметры загрузки:

IP : 3.89 .197 .203

26 апреля 2023 г., 15:04:46 


\section{ВЕРОЯТНОСТНОЕ ПРЕДСТАВЛЕНИЕ РЕШЕНИЯ НАЧАЛЬНО-КРАЕВОЙ ЗАДАЧИ ДЛЯ СИСТЕМЫ ПАРАБОЛИЧЕСКИХ УРАВНЕНИЙ}

Получено вероятностное представление решения начально-краевой задачи для системы параболических уравнений. На основе вероятностного представления определен случайных процесс, на траекториях которого строятся несмещенная и $\varepsilon$-смещенная оценки решения.

Ключевые слова и фразы: вероятностное представление, вероятности перехода, марковская цепь, статистическое моделирование.

1. Постановка задачи. В данной работе рассматривается начально-краевая задача для следующей системы параболических уравнений. Пусть $D-$ ограниченная область в $\mathbf{R}^{m}$ с достаточно гладкой границей $\partial D$ и $\Omega=D \times[0, T]-$ цилиндр в $\mathbf{R}^{m+1}$ с образующими, параллельными оси $t$, основанием которого служит область $D$ в плоскости $t=0$ ( $T$ - фиксированное число). Пусть заданы функции $y_{0 i}(x) \in C(\bar{D})$, $y_{i}(x, t) \in C(\partial D \times[0, T]), f_{i}(x, t) \in C(\bar{\Omega})$ и коэффициенты $a_{i}>0, c_{i j}>0(i, j=1, \ldots, n)$.

Рассмотрим начально-краевую задачу для следующей системы параболических уравнений:

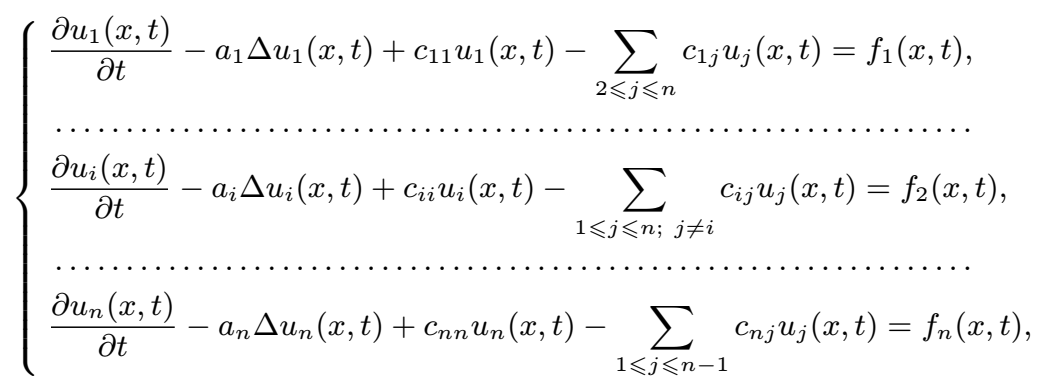

$(x, t) \in \Omega$, с начально-краевыми условиями

$$
\begin{cases}u_{i}(x, t)=y_{i}(x, t), & x \in \partial D, \quad t \in[0, T], \quad i=1, \ldots, n, \\ u_{i}(x, 0)=y_{0 i}(x), & x \in D, \quad i=1, \ldots, n\end{cases}
$$

В дальнейшем будем предполагать, что система (1) при начально-краевых условиях (2) имеет имеет единственное непрерывное решение

$$
u_{i}(x, t) \in C(\bar{D} \times[0, T]) \cap C^{2,1}(\bar{D} \times[0, T]) \quad(i=1, \ldots, n) .
$$

Вопросы разрешимости краевых задач для системы параболических уравнений общего вида исследованы в монографии [4] (см. также [5]).

В настоящей работе мы получаем вероятностное представление решения задачи (1)-(2) и с его помощью строим марковский случайный процесс, на траекториях которого можно определить несмещенную оценку в некоторой произвольной точке $(x, t) \in \Omega$. Для осуществления сказанного выписывается специальная система интегральных уравнений, связывающих значения функций $u_{i}(x, t)$ с их интегралами по

* Университет мировой экономики и дипломатии, Ташкент, Узбекистан; е-mail: raimova27@gmail.com 
сфероиду и шароиду с центром в точке $(x, t)$. Характеристической особенностью векторных вариантов метода Монте-Карло [6], [7], которые используются для решения систем интегральных уравнений, является матричный вес, который после каждого перехода в моделируемой цепи Маркова домножается на матрицу ядерных функций системы, поделенных на плотность переходных вероятностей. В настоящей работе для решения системы интегральных уравнений предлагается новый способ построения несмещенной оценки на траекториях моделируемой цепи Маркова. В отличие от классического векторного алгоритма метода Монте-Карло, в предложенной оценке не используются матричные веса, что значительно сокращает объем вычислительной работы.

Для построения вероятностного представления оценок используется формула параболического среднего для решения уравнения теплопроводности. Приведем известные факты, которые будут использованы в дальнейшем.

Как известно, фундаментальное решение $Z(x, t ; y, \tau)$ уравнения теплопроводности $u_{t}-a \Delta u=0$ имеет вид $Z(x, t ; y, \tau)=(4 \pi a(t-\tau))^{-m / 2} \exp \left(-|x-y|^{2} /(4 a(t-\tau))\right)$. С помощью фундаментального решения можно определить семейство областей $Q_{r}(x, t)$, зависящих от положительного параметра $r>0$ и точки $(x, t) \in \mathbf{R}^{m+1}$, полагая $Q_{r}(x, t)=\left\{(y, \tau): Z(x, t ; y, \tau)>(4 \pi a r)^{-m / 2}, \tau<t\right\}$. Область $Q_{r}(x, t)$, согласно [2], называют шароидом радиуса $r$ с центром в точке $(x, t)$, а границу $\partial Q_{r}(x, t)-$ сфероидом. Из определения $Q_{r}(x, t)$ следует, что шароид описывается следующим образом:

$$
Q_{r}(x, t)=\left\{(y, \tau):|x-y|^{2}<2 m a(t-\tau) \ln \frac{r}{t-r}, \tau<t\right\} .
$$

Каждое сечение шароида плоскостью $\tau=$ const представляет собой $m$-мерный шар $B(x, R(t-\tau))$ с центром в точке $x$ и радиусом $R(t-\tau)=\sqrt{2 m a(t-\tau) \ln (r /(t-r))}$. Пусть $(x, t) \in \Omega$ и $r=r(x, t)=\min \left\{R_{1}^{2}(x) e /(2 a m), t\right\}$, где $R_{1}(x)-$ расстояние от $x$ до границы области $D: R_{1}(x)=\inf \left\{\left|x-x^{\prime}\right|: x^{\prime} \in \partial D, x \in \bar{D}\right\}$. В этом случае $Q_{r}(x, t) \subset \bar{\Omega}$. С помощью фундаментального решения и формулы Грина осуществляется переход от системы дифференциальных уравнений к системе интегральных уравнений. Для этого в [10] доказано специальное соотношение, аналогичное приведенному в [3], связывающее значение функции $u(x, t)$ с ее интегралом по сфероиду и шароиду с центром в точке $(x, t)$. Резюмируем сказанное в виде следующей леммы.

Лемма 1 (о среднем значении). Пусть функиия $u(x, t)$ удовлетворяет уравнению $\partial u(x, t) / \partial t-a \Delta u(x, t)=f(x, t),(x, t) \in \Omega$. Тогда справедливо следующее соотношение:

$$
\begin{aligned}
u(x, t)= & a \iint_{\partial Q_{r}(x, t)}\left(1-\frac{t-\tau}{r}\right)\left(-\frac{\partial Z(x, t ; y, \tau)}{\partial n_{y}}\right) u(y, \tau) d s d \tau \\
& +\frac{1}{r} \iint_{Q_{r}(x, t)} Z_{r}(x, t ; y, \tau) u(y, \tau) d y d \tau+F_{r}(x, t)
\end{aligned}
$$

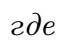

$$
\begin{aligned}
F_{r}(x, t) & =\frac{1}{r} \iint_{Q_{r}(x, t)}(r-(t-\tau)) Z_{r}(x, t ; y, \tau) f(y, \tau) d y d \tau \\
Z_{r}(x, t ; y, \tau) & =Z(x, t ; y, \tau)-(4 \pi a r)^{-m / 2}
\end{aligned}
$$

здесь $n_{y}$ - внешняя нормаль, $d s$ - элемент площади сферы $\partial B(x, R(t-\tau))$.

В следующем пункте эта лемма используется для получения специального интегрального представления функций $u_{i}(x, t)$. 
2. Преобразование системы (1) и интегральное представление. Определим семейство областей $Q_{r}^{(i)}(x, t)$, зависящих от положительного параметра $r>0$ и точки $(x, t) \in \mathbf{R}^{m+1}$, полагая $Q_{r}^{(i)}(x, t)=\left\{(y, \tau): Z_{r}^{(i)}(x, t ; y, \tau)>0, \tau<t\right\}$, где $Z_{r}^{(i)}(x, t ; y, \tau)$ определяется аналогично $Z_{r}(x, t ; y, \tau)$ заменой $a$ на $a_{i}$ (см. лемму 1$)$. Область $Q_{r}^{(i)}(x, t)$ назовем шароидом радиуса $r$ с центром в точке $(x, t)$, а границу $\partial Q_{r}^{(i)}(x, t)=\left\{(y, \tau): Z_{r}^{(i)}(x, t ; y, \tau)=0, \tau \leqslant t\right\}-$ сфероидом. Пусть $(x, t) \in \Omega$ и

$$
r=r(x, t)=\min \left\{\frac{R_{1}^{2}(x) e}{2 a_{1} m}, \ldots, \frac{R_{1}^{2}(x) e}{2 a_{n} m}, t\right\},
$$

где $R_{1}(x)$ - расстояние от $x$ до границы области $D$. В этом случае $Q_{r}^{(i)}(x, t) \subset \bar{\Omega}$. Применяя соотношение (3) к каждому из уравнений системы $(1)$, получим следующую систему интегральных уравнений $(i=1, \ldots, n)$ :

$$
\begin{aligned}
u_{i}(x, t)= & a_{i} \iint_{\partial Q_{r}^{(i)}(x, t)}\left(1-\frac{t-\tau}{r}\right)\left(-\frac{\partial Z^{(i)}(x, t ; y, \tau)}{\partial n_{y}}\right) u_{i}(y, \tau) d s d \tau \\
& +\frac{1}{r} \iint_{Q_{r}^{(i)}(x, t)}\left(1-(r-(t-\tau)) c_{i i}\right) Z_{r}^{(i)}(x, t ; y, \tau) u_{i}(y, \tau) d y d \tau \\
& +\frac{1}{r} \iint_{Q_{r}^{(i)}(x, t)}(r-(t-\tau)) Z_{r}^{(i)}(x, t ; y, \tau) \sum_{j=1, \ldots, n ; j \neq i} c_{i j} u_{j}(y, \tau) d y d \tau \\
& +\frac{1}{r} \iint_{Q_{r}^{(i)}(x, t)}(r-(t-\tau)) Z_{r}^{(i)}(x, t ; y, \tau) f_{i}(y, \tau) d y d \tau .
\end{aligned}
$$

Здесь

$$
\begin{aligned}
& Z^{(i)}(x, t ; y, \tau)=\left(4 \pi a_{i}(t-\tau)\right)^{-m / 2} \exp \left(-\frac{|x-y|^{2}}{4 a_{i}(t-\tau)}\right) \\
& Z_{r}^{(i)}(x, t ; y, \tau)=Z^{(i)}(x, t ; y, \tau)-\left(4 \pi a_{i} r\right)^{-m / 2} .
\end{aligned}
$$

3. Вероятностное представление решения задачи (1)-(2). После некоторых преобразований можно получить для первого слагаемого в правой части системы (4) следующее выражение:

$$
\begin{aligned}
I_{1}^{(i)}(x, t) & :=a_{i} \iint_{\partial Q_{r}^{(i)}(x, t)}\left(1-\frac{t-\tau}{r}\right)\left(-\frac{\partial Z^{(i)}(x, t ; y, \tau)}{\partial n_{y}}\right) u_{i}(y, \tau) d s d \tau \\
& =\left(1-q_{m}\right) \int_{0}^{\infty} q_{1}(\rho) d \rho \int_{S_{1}(0)} q_{2}(w) u_{i}\left(y^{(i)}(\rho, w), \tau(\rho)\right) d s \\
& =\left(1-q_{m}\right) \mathbf{E} u_{i}\left(y^{(i)}(\xi, \omega), \tau(\xi)\right),
\end{aligned}
$$

где

$$
\begin{aligned}
q_{1}(\rho) & =\rho^{m / 2} \exp (-\rho)\left(1-\exp \left(-\frac{2 \rho}{m}\right)\right)\left(\left(1-q_{m}\right) \Gamma\left(1+\frac{m}{2}\right)\right)^{-1} \\
q_{2}(w) & =\frac{1}{\sigma_{m}}=\Gamma\left(\frac{m}{2}\right)\left(2 \pi^{m / 2}\right)^{-1}, \quad q_{m}=\left(1+\frac{2}{m}\right)^{-(1+m / 2)} \\
y^{(i)}(\xi, \omega) & =x+\left(4 r \xi a_{i} \exp \left(-\frac{2 \xi}{m}\right)\right)^{1 / 2} \omega, \quad \tau(\xi)=t-r \exp \left(-\frac{2 \xi}{m}\right)
\end{aligned}
$$


$\xi-$ случайная величина, имеющая плотность распределения $q_{1}(\rho) ; \omega-$ случайная точка поверхности $S_{1}(0)$, имеющая плотность распределения $q_{2}(w), S_{1}(0)$ - единичная сфера; $d s$ - элемент поверхности, а $\sigma_{m}$ - площадь поверхности единичной сферы, $\Gamma(\cdot)$ — гамма-функция.

Рассмотрим последнее слагаемое в (4):

$$
\begin{aligned}
I_{2}^{(i)}(x, t):= & \frac{1}{r} \iint_{Q_{r}^{(i)}(x, t)}(r-(t-\tau)) Z_{r}^{(i)}(x, t ; y, \tau) f_{i}(y, \tau) d y d \tau \\
= & r q_{m} \int_{0}^{1} q_{3}(\nu) d \nu \int_{0}^{\infty} q_{4}(z) d z \int_{S_{1}(0)} d S_{w}\left(1-\nu^{2 / m} \exp \left(\frac{-2 z}{m+2}\right)\right) \\
& \times f_{i}\left(y_{1}^{(i)}(z, \nu, w), \tau_{1}(z, \nu)\right) \\
= & r q_{m} \mathbf{E}\left\{\left(1-v^{2 / m} \exp \left(\frac{-2 \xi_{1}}{m+2}\right)\right) f_{i}\left(y_{1}^{(i)}\left(\xi_{1}, v, \omega\right), \tau_{1}\left(\xi_{1}, v\right)\right)\right\}
\end{aligned}
$$

где $q_{3}(\nu)=(1-\nu) \nu^{2 / m-1}(\mathrm{~B}(2,2 / m))^{-1}$ - плотность бета-распределения с параметрами $(2,2 / m), \mathrm{B}(\cdot, \cdot)$ - бета-функция, $q_{4}(z)=\exp (-z) z^{m / 2-1}(\Gamma(m / 2))^{-1}-$ плотность гамма-распределения с параметром $m / 2, \omega-$ случайный единичный вектор,

$$
\begin{aligned}
y_{1}^{(i)}\left(\xi_{1}, v, \omega\right) & =x+\left(\frac{4 m}{m+2} r a_{i} \xi_{1} v^{2 / m} \exp \left(-\frac{2 \xi_{1}}{m+2}\right)\right)^{1 / 2} \omega \\
\tau_{1}\left(\xi_{1}, v\right) & =t-r v^{2 / m} \exp \left(-\frac{2 \xi_{1}}{m+2}\right)
\end{aligned}
$$

$\xi_{1}$ - случайная величина с плотностью распределения $q_{4}(z), v-$ случайная величина с плотностью распределения $q_{3}(\nu)$.

Пусть

$$
r=r(x, t)=\min \left\{\frac{e R_{1}^{2}(x)}{2 m a_{1}}, \ldots, \frac{e R_{1}^{2}(x)}{2 m a_{n}} ; \frac{1}{c_{11}}, \ldots, \frac{1}{c_{n n}} ; t\right\}
$$

тогда $\overline{Q_{r}^{(i)}}(x, t) \in \Omega$ и функции

$$
p_{1}^{(i)}(x, t ; y, \tau)=\frac{\left(1-(r-(t-\tau)) c_{i}\right) Z_{r}^{(i)}(x, t ; y, \tau}{r q_{m}\left(1-r q_{1 m} c_{i i}\right)} \mathbf{I}\left\{(y, \tau) \in Q_{r}^{(i)}(x, t)\right\}
$$

являются плотностями распределений в $Q_{r}^{(i)}(x, t)$ при фиксированных $(x, t)$. Здесь

$$
q_{1 m}=1-\frac{1}{2}\left(\frac{m+2}{m+4}\right)^{1+m / 2}
$$

Пусть $\left(y_{2}^{(i)}, \tau_{2}^{(i)}\right)$ - случайная точка шароида $Q_{r}^{(i)}(x, t)$, имеющая при фиксированных $(x, t)$ плотность распределения $p_{1}^{(i)}(x, t ; y, \tau)(i=1, \ldots, n)$. Тогда

$$
\begin{gathered}
\frac{1}{r} \iint_{Q_{r}^{(i)}(x, t)}\left(1-(r-(t-\tau)) c_{i i}\right) Z_{r}^{(i)}(x, t ; y, \tau) u_{i}(y, \tau) d y d \tau \\
=q_{m}\left(1-r c_{i i} q_{1 m}\right) \mathbf{E} u_{i}\left(y_{2}^{(i)}, \tau_{2}^{(i)}\right) \quad(i=1, \ldots, n)
\end{gathered}
$$

Подставляя полученные результаты в (4), получим вероятностное представление, позволяющее построить оценку решения задачи (1)-(2). Итак, справедлива следующая теорема. 
Теорема 1. Для решения системы (1) справедливо следующее вероятностное представление: для $i=1, \ldots, n$

$$
\begin{aligned}
& u_{i}(x, t)=\left(1-q_{m}\right) \mathbf{E} u_{i}\left(y^{(i)}(\xi, \omega), \tau(\xi)\right)+q_{m}\left(1-r c_{i i} q_{1 m}\right) \mathbf{E} u_{i}\left(y_{2}^{(i)}, \tau_{2}^{(i)}\right) \\
& +q_{m} r \mathbf{E}\left\{\left(1-v^{2 / m} \exp \left(-\frac{2 \xi_{1}}{m+2}\right)\right) \sum_{j=1, \ldots, n ; j \neq i} c_{i j} u_{j}\left(y_{1}^{(i)}\left(\xi_{1}, v, \omega\right), \tau_{1}\left(\xi_{1}, v\right)\right)\right\} \\
& +q_{m} r \mathbf{E}\left\{\left(1-v^{2 / m} \exp \left(-\frac{2 \xi_{1}}{m+2}\right)\right) f_{i}\left(y_{1}^{(i)}\left(\xi_{1}, v, \omega\right), \tau_{1}\left(\xi_{1}, v\right)\right)\right\},
\end{aligned}
$$

где $y^{(i)}(\xi, \omega)$ u $\tau(\xi)$ определяются формулами $(5)$, a $y_{1}^{(i)}\left(\xi_{1}, v, \omega\right) u \tau_{1}\left(\xi_{1}, v\right)-\oint о р м у$ лами (6).

Далее в соответствии с представлением (8) строится случайный процесс в фазовом пространстве $\Omega$ и предлагается алгоритм моделирования.

4. Описание случайного процесса и способ моделирования. Пусть $r=$ $r(x, t)$ определено формулой (7). Тогда функции

$$
\begin{aligned}
& p_{0}^{(i)}(x, t ; y, \tau)=\frac{1-(t-\tau) / r}{1-q_{m}}\left(-\frac{\partial Z^{(i)}(x, t ; y, \tau)}{\partial n_{y}}\right) \mathbf{I}\left\{(y, \tau) \in Q_{r}^{(i)}(x, t)\right\} \\
& p_{1}^{(i)}(x, t ; y, \tau)=\frac{\left(1-(r-(t-\tau)) c_{i i}\right) Z_{r}^{(i)}(x, t ; y, \tau)}{r q_{m}\left(1-r q_{1 m} c_{i i}\right)} \mathbf{I}\left\{(y, \tau) \in Q_{r}^{(i)}(x, t)\right\} \\
& p_{2}^{(i)}(x, t ; y, \tau)=\frac{Z_{r}^{(i)}(x, t ; y, \tau)}{r q_{m}} \mathbf{I}\left\{(y, \tau) \in Q_{r}^{(i)}(x, t)\right\}
\end{aligned}
$$

являются плотностями распределений в $\overline{Q_{r}^{(i)}}(x, t)$ при фиксированных $(x, t)(i=$ $1, \ldots, n)$. Определим в $\Omega$ случайный процесс следующим образом. Зададим матрицу переходных вероятностей

$$
A(x, t)=\left(\begin{array}{cccc}
\alpha_{11} & \alpha_{12} & \cdots & \alpha_{1(n+1)} \\
\alpha_{21} & \alpha_{22} & \cdots & \alpha_{2(n+1)} \\
\cdots & \cdots & \cdots & \cdots \\
\alpha_{n 1} & \alpha_{n 2} & \cdots & \alpha_{n(n+1)} \\
0 & 0 & \cdots & 1
\end{array}\right)
$$

где

здесь

$$
\begin{gathered}
\alpha_{i i}=1-q_{m} c_{i i} q_{1 m} r(x, t), \quad \alpha_{i j}=\frac{\beta_{i} c_{i j}}{M_{i}} \quad(i, j=1, \ldots, n, i \neq j), \\
\alpha_{i(n+1)}=\frac{q_{m} c_{i i} q_{1 m} r(x, t)}{n} \quad(i=1, \ldots, n),
\end{gathered}
$$

$$
\beta_{i}=\frac{q_{m} c_{i i} q_{1 m} r(x, t)(n-1)}{n}, \quad M_{i}=\sum_{j=1, \ldots, n ; j \neq i} c_{i j} .
$$

Определим матрицу плотностей переходных вероятностей $P(x, t ; y, \tau)$ :

$$
P(x, t ; y, \tau)=\left(\begin{array}{cccc}
p_{11}(x, t ; y, \tau) & p_{12}(x, t ; y, \tau) & \ldots & p_{1(n+1)}(x, t ; y, \tau) \\
p_{21}(x, t ; y, \tau) & p_{22}(x, t ; y, \tau) & \ldots & p_{2(n+1)}(x, t ; y, \tau) \\
\ldots & \ldots & \ldots & \ldots \\
p_{n 1}(x, t ; y, \tau) & p_{n 2}(x, t ; y, \tau) & \ldots & p_{n(n+1)}(x, t ; y, \tau) \\
0 & 0 & \ldots & 1
\end{array}\right)
$$




$$
\begin{gathered}
p_{i j}(x, t ; y, \tau)=p_{2}^{(i)}(x, t ; y, \tau)(i=1, \ldots, n, j=\overline{1, n+1}, i \neq j) \text { и для } i=1, \ldots, n \\
p_{i i}(x, t ; y, \tau)=\frac{\left(1-q_{m}\right) p_{0}^{(i)}(x, t ; y, \tau)+q_{m}\left(1-r q_{1 m} c_{i i}\right) p_{1}^{(i)}(x, t ; y, \tau)}{1-r(x, t) q_{1 m} c_{i i} q_{m}} .
\end{gathered}
$$

Далее фиксируем начальную точку $\left(x_{0}, t_{0}\right)=(x, t)$ и номер уравнения $i_{0} \in\{1, \ldots, n\}$. Пусть в начальный момент частица находится в точке $\left(x_{0}, t_{0}\right)=(x, t)$. За один шаг осуществляется переход $i_{k} \rightarrow i_{k+1}$ в соответствии с матрицей переходных вероятностей $A\left(x_{k}, t_{k}\right)$, т.е. с вероятностью $\alpha_{i_{k} i_{k+1}}\left(x_{k}, t_{k}\right)$ частица переходит из точки $\left(x_{k}, t_{k}\right)$ в точку $\left(x_{k+1}, t_{k+1}\right)$, имеющую плотность распределения $p_{i_{k} i_{k+1}}\left(x_{k}, t_{k} ; y, \tau\right)$. Вероятность обрыва траектории в точке $\left(x_{n}, t_{n}\right)$ равна

$$
g\left(x_{n}, t_{n}\right)= \begin{cases}1, & \left(x_{n}, t_{n}\right) \in \partial \Omega \\ \alpha_{i_{n-1} n+1}\left(x_{n-1}, t_{n-1}\right), & \left(x_{n}, t_{n}\right) \in \Omega\end{cases}
$$

Последовательность координат частицы определяется следующим образом.

а) Если плотность распределения точки $\left(x_{n+1}, t_{n+1}\right)$ равна $p_{0}^{(i)}\left(x_{n}, t_{n} ; y, \tau\right)$ при фиксированных $\left(x_{n}, t_{n}\right)$, то

$$
\left\{\begin{array}{l}
x_{n+1}=x_{n}+2\left(r\left(x_{n}, t_{n}\right) \xi_{n} a_{i}\right)^{1 / 2} \exp \left(-\frac{\xi_{n}}{m}\right) \omega_{n} \\
t_{n+1}=t_{n}-r\left(x_{n}, t_{n}\right) \exp \left(-\frac{2 \xi_{n}}{m}\right)
\end{array}\right.
$$

где $\left\{\xi_{n}\right\}_{n=0}^{\infty}$ и $\left\{\omega_{n}\right\}_{n=0}^{\infty}$ - последовательности независимых случайных величин с плотностью распределения $q_{1}(\rho)$ и независимых изотропных векторов соответственно. Значение $r\left(x_{n}, t_{n}\right)$ определяется по формуле (7).

b) Если плотность распределения точки $\left(x_{n+1}, t_{n+1}\right)$ равна $p_{1}^{(i)}\left(x_{n}, t_{n} ; y, \tau\right)$ при фиксированных $\left(x_{n}, t_{n}\right)$, то

$$
\left\{\begin{array}{l}
x_{n+1}=x_{n}+2\left(\frac{m}{m+2} r\left(x_{n}, t_{n}\right) \xi_{n}^{\prime}\left(v_{n}^{\prime}\right)^{2 / m} a_{i} \exp \left(-\frac{2 \xi_{n}^{\prime}}{m+2}\right)\right)^{1 / 2} \omega_{n} \\
t_{n+1}=t_{n}-r\left(x_{n}, t_{n}\right)\left(v_{n}^{\prime}\right)^{2 / m} \exp \left(-\frac{2 \xi_{n}^{\prime}}{m+2}\right)
\end{array}\right.
$$

где $\left\{\xi_{n}^{\prime}\right\}_{n=0}^{\infty}$ и $\left\{v_{n}^{\prime}\right\}_{n=0}^{\infty}-$ последовательности независимых случайных величин, полученных по следующему алгоритму (использован метод исключения):

I) моделируются $\xi$ - гамма-распределенная случайная величина с параметром $m / 2, \gamma$ - равномерно распределенная на отрезке $(0,1), v$ - бета-распределенная случайная величина с параметрами $(2,2 / m)$;

II) если $\gamma>1-c_{i i} r\left(1-v^{2 / m} \exp (-2 \xi /(m+2))\right)$, то выполняется I), и т.д.; в противном случае полагаем $v^{\prime}=v, \xi^{\prime}=\xi$.

c) Если плотность распределения точки $\left(x_{n+1}, t_{n+1}\right)$ равна $p_{2}^{(i)}\left(x_{n}, t_{n} ; y, \tau\right)$ при фиксированных $\left(x_{n}, t_{n}\right)$, то

$$
\left\{\begin{array}{l}
x_{n+1}=x_{n}+2\left(\frac{m}{m+2} r\left(x_{n}, t_{n}\right) \zeta_{n} v_{n}^{2 / m} a_{i} \exp \left(-\frac{2 \zeta_{n}}{m+2}\right)\right)^{1 / 2} \omega_{n} \\
t_{n+1}=t_{n}-r\left(x_{n}, t_{n}\right) v_{n}^{2 / m} \exp \left(-\frac{2 \zeta_{n}}{m+2}\right)
\end{array}\right.
$$

где $\left\{\zeta_{n}\right\}_{n=0}^{\infty}, \quad\left\{v_{n}\right\}_{n=0}^{\infty}$ и $\left\{\omega_{n}\right\}_{n=0}^{\infty}$ - последовательности независимых гаммараспределенных случайных величин с параметром $m / 2$, бета-распределенных случайных величин с параметрами $(2,2 / m)$ и независимых изотропных векторов соответственно. 
В случае, если в момент времени $n$ произошел обрыв, полагаем $\left(x_{n+k}, t_{n+k}\right)=$ $\left(x_{n}, t_{n}\right), k=0,1,2, \ldots$. Очевидно, что последовательность координат частицы образует марковскую цепь. Случайный процесс, подобный описанному выше, был предложен в работе [1] для решения краевой задачи для уравнения теплопроводности. В работе [3] аналогичный случайный процесс построен для уравнения теплопроводности с переменным коэффициентом поглощения.

В дальнейшем нам понадобится следующее вспомогательное утверждение.

Лемма 2. С вероятностью 1 марковская иепь $\left\{x_{n}, t_{n}\right\}_{n=0}^{\infty}$ сходится при $n \rightarrow \infty$ $\kappa$ случайной точке граничь $\left(x_{\infty}, t_{\infty}\right) \in \partial \Omega$ либо обрывается внутри области.

Д о к а з а т е л ь с т в о. Так как $\left\{t_{n}\right\}$ - убывающая последовательность и $t_{n} \geqslant 0$, то при $n \rightarrow \infty$ она имеет предел $t_{\infty}=\lim _{n \rightarrow \infty} t_{n}$. Пусть $\mathfrak{R}_{n}$ есть $\sigma$-алгебра, порожденная случайными величинами $\left\{\omega_{k}\right\}_{k=0}^{n-1},\left\{v_{k}\right\}_{k=0}^{n-1},\left\{\xi_{k}\right\}_{k=0}^{n-1},\left\{v_{k}^{\prime}\right\}_{k=0}^{n-1},\left\{\xi_{k}^{\prime}\right\}_{k=0}^{n-1}$. Из определения $\mathfrak{R}_{n}$ и формул (9)-(11) следует, что $x_{n}$ измерима относительно $\mathfrak{R}_{n}$. Координаты векторного процесса образуют ограниченный мартингал относительно $\left\{\Re_{m}\right\}_{m=1}^{\infty}$ :

$$
\begin{aligned}
\mathbf{E} & \left.x_{n+1} \mid \mathfrak{R}_{n}\right)=\mathbf{E}\left\{\left(1-q_{m}\right)\left[x_{n}+2\left(r\left(x_{n}, t_{n}\right) \xi_{n} a_{i_{n}}\right)^{1 / 2} \exp \left(-\frac{\xi_{n}}{m}\right) \omega_{n}\right]\right. \\
& +q_{m}\left(1-q_{1 m} r\left(x_{n}, t_{n}\right) c_{i_{n} i_{n}}\right) \\
& \times\left[x_{n}+2\left(\frac{m}{m+2} r\left(x_{n}, t_{n}\right) a_{i_{n}} \xi_{n}^{\prime}\left(v_{n}^{\prime}\right)^{2 / m}\right)^{1 / 2} \exp \left(-\frac{\xi_{n}^{\prime}}{m+2}\right) \omega_{n}\right] \\
& +\frac{n-1}{n} c_{i_{n} i_{n}} r\left(x_{n}, t_{n}\right) q_{m} q_{1 m} \\
& \times\left[x_{n}+2\left(\frac{m}{m+2} r\left(x_{n}, t_{n}\right) a_{i_{n}} \zeta_{n} v_{n}^{2 / m}\right)^{1 / 2} \exp \left(-\frac{\zeta_{n}}{m+2}\right) \omega_{n}\right] \\
& \left.+\frac{c_{i_{n} i_{n}} r\left(x_{n}, t_{n}\right) q_{m} q_{1 m}}{n} x_{n} \mid \mathfrak{R}_{n}\right\} \\
= & \left(1-q_{m}\right)\left[x_{n}+2\left(r\left(x_{n}, t_{n}\right) a_{i_{n}}\right)^{1 / 2} \mathbf{E}\left(\xi_{n}^{1 / 2} \exp \left(-\frac{\xi_{n}}{m}\right) \omega_{n}\right)\right] \\
& +q_{m}\left(1-q_{1 m} c_{i_{n} i_{n}} r\left(x_{n}, t_{n}\right)\right) \\
& \times\left[x_{n}+2\left(\frac{m}{m+2} r\left(x_{n}, t_{n}\right) a_{i_{n}}\right)^{1 / 2} \mathbf{E}\left(\left(\xi_{n}^{\prime}\left(v_{n}^{\prime}\right)^{2 / m}\right)^{1 / 2} \exp \left(-\frac{\xi_{n}^{\prime}}{m+2}\right) \omega_{n}\right)\right] \\
& +\frac{n-1}{n} c_{i_{n} i_{n}} r\left(x_{n}, t_{n}\right) q_{m} q_{1 m} \\
& \times\left[x_{n}+2\left(\frac{m}{m+2} r\left(x_{n}, t_{n}\right) a_{i_{n}}\right)^{1 / 2} \mathbf{E}\left(\left(\zeta_{n} v_{n}^{2 / m}\right)^{1 / 2} \exp \left(-\frac{\zeta_{n}}{m+2}\right) \omega_{n}\right)\right] \\
& +\frac{c_{i_{n} i_{n}} r\left(x_{n}, t_{n}\right) q_{m} q_{1 m}}{n} x_{n} \\
& \left(1-q_{m}\right) x_{n}+q_{m}\left(1-q_{1 m} c_{i_{n} i_{n}} r\left(x_{n}, t_{n}\right)\right) x_{n}+c_{i_{n} i_{n}} r\left(x_{n}, t_{n}\right) q_{m} q_{1 m} x_{n}=x_{n} .
\end{aligned}
$$

Поскольку последовательность $\left\{x_{n}\right\}$ является ограниченным мартингалом, то она сходится с вероятностью единица [9]. Пусть $\left(x_{\infty}, t_{\infty}\right)=\lim _{n \rightarrow \infty}\left(x_{n}, t_{n}\right)-$ предельный вектор. Нам остается показать, что если процесс не оборвется внутри области, то он обязательно выйдет на границу области. Предположим, что обрыва внутри области за конечное число шагов не произошло. Покажем, что $\left(x_{\infty}, t_{\infty}\right) \in \partial \Omega$. На самом деле, если $t_{\infty}=0$, то это очевидно. Пусть $t_{\infty}>0$. Так как процесс $x_{n}$ сходится, то 
$\mathbf{E}_{\left(x_{0}, t_{0}\right)}\left|x_{n+1}-x_{n}\right| \rightarrow 0$. Согласно формулам (9)-(11), имеет место равенство

$$
\mathbf{E}_{\left(x_{0}, t_{0}\right)}\left|x_{n+1}-x_{n}\right|=\mathbf{E}_{\left(x_{0}, t_{0}\right)}\left\{\sqrt{r\left(x_{n}, t_{n}\right)} h\left(r\left(x_{n}, t_{n}\right)\right)\right\} .
$$

Здесь функция $h(r)$ строго положительна. Применяя теорему Лебега об ограниченной сходимости, получаем $\mathbf{E}_{\left(x_{0}, t_{0}\right)}\left(r\left(x_{\infty}, t_{\infty}\right)\right)^{1 / 2}=0$. Значит, $r\left(x_{\infty}, t_{\infty}\right)=0$ п. н. Тогда из определения $r(x, t)$ (см. (7)) следует равенство $R_{1}\left(x_{\infty}\right)=0$, т.е. $\left(x_{\infty}, t_{\infty}\right) \in \partial \Omega$. Лемма доказана.

5. Построение несмещенной и $\varepsilon$-смещенной оценок решения. Пусть $\left(x_{k}, t_{k}\right)_{k=0}^{\infty}$ - траектория случайного процесса, описанного выше, и

$$
\Theta_{0}=1, \quad \Theta_{n}=\Theta_{n-1} V_{i_{n-1} i_{n}}\left(x_{n-1}, t_{n-1} ; x_{n}, t_{n}\right)
$$

где $V_{i j}\left(x_{n-1}, t_{n-1} ; x_{n}, t_{n}\right)$ определяются равенствами: для $i, j=1, \ldots, n, i \neq j$

$$
\begin{aligned}
V_{i j}\left(x_{n-1}, t_{n-1} ; x_{n}, t_{n}\right) & =\frac{n M_{i}}{(n-1) c_{i i} q_{1 m}}\left(1-v_{n}^{2 / m} \exp \left(-\frac{2 \zeta_{n}}{m+2}\right)\right), \\
V_{i i}\left(x_{n-1}, t_{n-1} ; x_{n}, t_{n}\right) & =1, \\
V_{i(n+1)}\left(x_{n-1}, t_{n-1} ; x_{n}, t_{n}\right) & =\frac{n}{c_{i i} q_{1 m}}\left(1-v_{n}^{2 / m} \exp \left(-\frac{2 \zeta_{n}}{m+2}\right)\right) .
\end{aligned}
$$

Здесь $\left\{\zeta_{n}\right\}_{n=0}^{\infty}$ и $\left\{v_{n}\right\}_{n=0}^{\infty}-$ последовательности независимых гамма-распределенных случайных величин с параметром $m / 2$ и бета-распределенных случайных величин с параметрами $(2,2 / m)$ соответственно. Определим последовательность $\left\{\eta_{n}\left(i_{0}\right)\right\}$ :

$$
\eta_{n}\left(i_{0}\right)=\Theta_{n} F\left(x_{n}, t_{n}\right)=\Theta_{n} \times \begin{cases}u_{j}\left(x_{n}, t_{n}\right), & i_{n}=j, \quad j \neq n+1, \\ f_{i_{n-1}}\left(x_{n}, t_{n}\right), & i_{n}=n+1 .\end{cases}
$$

Если в момент времени $n$ произошел обрыв, то положим $\eta_{n+k}\left(i_{0}\right)=\eta_{n}\left(i_{0}\right)$, $\left(x_{n+k}, t_{n+k}\right)=\left(x_{n}, t_{n}\right), k=1,2, \ldots$. Пусть $\mathfrak{R}_{n}$ есть $\sigma$-алгебра, порожденная течением процесса до момента $n$ включительно. Справедлива следующая теорема.

Теорема 2. Последовательность $\left\{\eta_{n}\left(i_{0}\right)\right\}_{n=1}^{\infty}$ образует мартингал относительно $\Re_{n}$. Если

$$
\begin{gathered}
\sum_{j=1, \ldots, n ; j \neq i} c_{i j}<\frac{n-1}{n} c_{i i} q_{1 m} \quad(i=1, \ldots, n), \\
\max _{(x, t) \in \Omega}\left|f_{i}(x, t)\right| \leqslant c_{0} \quad\left(c_{0}=\text { const }, i=1, \ldots, n\right),
\end{gathered}
$$

mо $\left\{\eta_{n}\left(i_{0}\right)_{n=1}^{\infty}\right\}-$ равномерно интегрируемый мартингал.

Д о к а з а т е л ь с т в о. По построению величина $\eta_{n}\left(i_{0}\right)$ является $\mathfrak{R}_{n}$ измеримой. Далее,

$$
\begin{aligned}
& \mathbf{E}\left(\eta_{n+1}\left(i_{0}\right) \mid \mathfrak{R}_{n}\right)=\mathbf{E}\left(\Theta_{n+1} F\left(x_{n+1}, t_{n+1}\right) \mid \mathfrak{R}_{n}\right) \\
& =\mathbf{E}\left(\Theta_{n} V_{i_{n} i_{n+1}}\left(x_{n}, t_{n} ; x_{n+1}, t_{n+1}\right) F\left(x_{n+1}, t_{n+1}\right) \mid \mathfrak{R}_{n}\right) \\
& =\Theta_{n} \mathbf{E}\left(V_{i_{n} i_{n+1}}\left(x_{n}, t_{n} ; x_{n+1}, t_{n+1}\right) F\left(x_{n+1}, t_{n+1}\right)\right) \\
& =\Theta_{n}\left(\sum_{j=1, \ldots, n} \alpha_{i_{n} j} \iint_{Q_{r}^{\left(i_{n}\right)}\left(x_{n}, t_{n}\right)} p_{i_{n} j}\left(x_{n}, t_{n} ; y, \tau\right) u_{j}(y, \tau) d y d \tau\right. \\
& \left.\quad+\alpha_{i_{n}(n+1)} \iint_{Q_{r}^{\left(i_{n}\right)}\left(x_{n}, t_{n}\right)} p_{i_{n}(n+1)}\left(x_{n}, t_{n} ; y, \tau\right) f_{i_{n}}(y, \tau) d y d \tau\right)=\eta_{n}\left(i_{0}\right) .
\end{aligned}
$$


Таким образом, последовательность $\left\{\eta_{n}\left(i_{0}\right)\right\}$ является мартингалом. Чтобы доказать равномерную интегрируемость $\left\{\eta_{n}\left(i_{0}\right)\right\}$, достаточно показать, что $\left|\eta_{n}\left(i_{0}\right)\right| \leqslant C<$ $\infty$. Так как $u_{i}(x, t) \in C(\bar{D} \times[0, T]) \cap C^{2,1}(\bar{D} \times[0, T])$ и $\bar{\Omega}$ - ограниченная область, то $\left|u_{i}(x, t)\right| \leqslant C_{1}<\infty$ для $(x, t) \in \bar{\Omega}$. В силу условий теоремы $\left|\Theta_{n}\right| \leqslant 1$ и, следовательно, $\left|\eta_{n}\left(i_{0}\right)\right| \leqslant C_{2}<\infty$. Из последнего вытекает, что последовательность $\left\{\eta_{n}\left(i_{0}\right)\right\}$ является равномерно интегрируемой. Теорема доказана.

Теперь исследуем вопрос о реализуемости оценки $\eta_{n}\left(i_{0}\right)$. Рассмотрим несколько видоизмененный процесс с траекториями меньшей длины и оценки на нем со сколь угодно малым смещением. Выберем $\varepsilon$ достаточно малым и рассмотрим внутреннюю $\varepsilon$-окрестность границы $(\partial \Omega)_{\varepsilon}=\{D \times[0, \varepsilon]\} \cup\left\{(\partial D)_{\varepsilon} \times[0, T]\right\}$.

Пусть $N_{1}$ - момент обрыва процесса внутри области и $N_{\varepsilon}$ - момент первого попадания в $\varepsilon$-окрестность границы $(\partial \Omega)_{\varepsilon}$. Определим момент остановки $N=$ $\min \left\{N_{1}, N_{\varepsilon}\right\}$ процесса $\left\{\left(x_{n}, t_{n}\right)\right\}$ следующим образом. Тогда вероятность обрыва траектории в точке $\left\{\left(x_{n}, t_{n}\right)\right\}$ будет равна

$$
g\left(x_{n}, t_{n}\right)= \begin{cases}1, & \left(x_{n}, t_{n}\right) \in(\partial \Omega)_{\varepsilon}, \\ \alpha_{i_{n-1}(n+1)}\left(x_{n-1}, t_{n-1}\right), & \left(x_{n}, t_{n}\right) \in \bar{\Omega} \backslash(\partial \Omega)_{\varepsilon} .\end{cases}
$$

Из леммы 2 следует, что $N<\infty$. Непосредственной проверкой можно убедиться в том, что процесс $\left\{\left(x_{n}, t_{n}\right)\right\}$ удовлетворяет свойству мажорирования. Тогда, согласно теореме о мажорируемых процессах, математическое ожидание момента остановки $N$ конечно (см. [3]).

Теорема 3. Пусть выполнень условия теоремь 2. Тогда $\eta_{N}\left(i_{0}\right)$ является несмещенной оченкой, имеющей конечную дисперсию, для $u_{i_{0}}(x, t)-i_{0}-\breve{u}$ компоненть вектора решения $u(x, t)$ системь $(1)$.

Д о к а з а т е л ь с т в о. Поскольку последовательность $\left\{\eta_{n}\left(i_{0}\right)\right\}$ является равномерно интегрируемым мартингалом и $N$ - марковский момент, то, согласно теореме Дуба о преобразовании свободного выбора (см. [8], [9]), выполнено равенство $\mathbf{E}_{\left(x_{0}, t_{0}\right)} \eta_{N}\left(i_{0}\right)=\mathbf{E}_{\left(x_{0}, t_{0}\right)} \eta_{1}\left(i_{0}\right)$. Из определения $\eta_{1}\left(i_{0}\right)$ следует, что $\mathbf{E}_{\left(x_{0}, t_{0}\right)} \eta_{1}\left(i_{0}\right)=$ $u_{i_{0}}(x, t)$. В силу условий теоремы 2 справедливо $\mathbf{E}\left(\eta_{N}\left(i_{0}\right)\right)^{2}<\infty$ и, следовательно, дисперсия оценки $\eta_{N}\left(i_{0}\right)$ конечна. Теорема доказана.

Далее, по $\eta_{N}\left(i_{0}\right)$ строим стандартным способом смещенную, но практически реализуемую на ЭВМ оценку $\eta_{N}^{*}\left(i_{0}\right)$. Пусть $\Psi_{i}(x, t)=y_{i}(x, t)$ при $x \in \partial D, t \in[0, T]$ и $\Psi_{i}(x, 0)=y_{0 i}(x)$ при $x \in \bar{D}$, и пусть $\left(x^{*}, t^{*}\right)$ - ближайшая к $(x, t)$ точка границы $\partial \Omega$. Тогда $\eta_{N}^{*}$ получается заменой $u_{i}\left(x_{N}, t_{N}\right)$ в $\eta_{N}\left(i_{0}\right)$ на $\Psi_{i}\left(x_{N}^{*}, t_{N}^{*}\right)$. Оценим смещение оценки $\eta_{N}^{*}\left(i_{0}\right)$. Ясно, что $\left|\mathbf{E}_{(x, t)} \eta_{N}\left(i_{0}\right)-u_{i_{0}}(x, t)\right| \leqslant \mathbf{E}_{(x, t)}\left|\eta_{N}^{*}\left(i_{0}\right)-\eta_{N}\left(i_{0}\right)\right|$. Если $N=N_{1}$, то процесс обрывается, не попадая в $(\partial \Omega)_{\varepsilon}$, и $\eta_{N}^{*}\left(i_{0}\right)=\eta_{N}\left(i_{0}\right)$. Если же $N=N_{\varepsilon}$, то $\left(x_{N}, t_{N}\right) \in(\partial \Omega)_{\varepsilon}$. Пусть $A_{i}(\varepsilon)$ - модуль непрерывности функции $u_{i}(x, t)$. Тогда справедливо неравенство $\left|\eta_{N}^{*}\left(i_{0}\right)-\eta_{N}\left(i_{0}\right)\right| \leqslant\left|\Theta_{N}\right| A(\varepsilon)$, где $A(\varepsilon)=\max _{i}\left\{A_{i}(\varepsilon)\right\}$. Так как $\left|\Theta_{N}\right| \leqslant 1$, то $\mathbf{E}_{(x, t)}\left|\eta_{N}^{*}\left(i_{0}\right)-u_{i_{0}}(x, t)\right| \leqslant A(\varepsilon)$. Конечность дисперсии следует из того,что $\mathbf{E} \eta_{N}^{2}\left(i_{0}\right)<\infty$.

Автор выражает глубокую благодарность рецензенту за замечания, которые способствовали улучшению изложения и устранению неточностей.

\section{СПИСОК ЛИТЕРАТУРЫ}

1. Ермаков C. М., Некруткин В. B., Сипин A. С. Случайные процессы для решения классических уравнений математической физики. М.: Наука, 1984, 205 с.

2. Купчов Л. П. Свойства среднего и принцип максимума для параболических уравнений второго порядка. - Докл. АН СССР, 1978, т. 242, № 3, с. 529-532. 
3. Курбанмурадов О. Методы блуждания по шароидам для решения уравнения теплопроводности. - Теория и алгоритмы статистического моделирования. Новосибирск: ВЦ СО АН СССР, 1984, с. 67-77.

4. Ладыженская О.А., Солонников В.А., Уральчева Н. Н. Линейные и квазилинейные уравнения параболического типа. М.: Наука, 1967, 736 с.

5. Лионс Ж.-Л. Оптимальное управление системами, описываемыми уравнениями с частными производными. М.: Мир, 1972, 414 с.

6. Михайлов Г.А. Оптимизация весовых методов Монте-Карло. М.: Наука, 1987, $239 \mathrm{c}$.

7. Михайлов Г.А. Весовые методы Монте-Карло. Новосибирск: Изд-во СО РАН, $2000,248 \mathrm{c}$.

8. Мейер П.-А. Вероятность и потенциалы. М.: Мир, 1973, 324 с.

9. Ширяев А. Н. Вероятность. М.: Наука, 1989, 640 с.

10. Rasulov A., Mascagni M., Raimova G. Monte Carlo Methods for the Solution of Linear and Nonlinear Boundary Value Problems. Tashkent: UWED, 2006, 346 p.

Поступила в редакцию

18.VI.2009

Исправленный вариант 20.X.2012

(c) 2012 г.

РОЗОВСКИЙ Л. В.*

\section{ОБ УСЛОВИЯХ СХОДИМОСТИ ОБОБЩЕННОГО РЯДА СПИЦЕРА}

\footnotetext{
В заметке приведены оптимальные моментные условия, при которых сходится обобщенный ряд Спицера.

Ключевые слова и фразы: суммы независимых случайных величин, ряд Спицера, нормальное распределение.
}

Рассмотрим независимые одинаково распределенные случайные величины $X, X_{1}, X_{2}, \ldots$ с нулевым средним и конечной положительной дисперсией $\sigma^{2}$. Положим $S_{n}=X_{1}+\cdots+X_{n}$.

Мы приведем один результат, связанный с оценкой величины

$$
\mathbf{P}\left(S_{n}<0\right)-\frac{1}{2}
$$

а именно сформулируем оптимальные условия, при которых сходится ряд

$$
\sum_{n \geqslant 1} \frac{g(n)}{n}\left|\mathbf{P}\left(S_{n}<0\right)-\frac{1}{2}\right|,
$$

где положительные веса $g(n)$ имеют степенной характер.

При $g(n) \equiv 1$ ряд из (1) аналогичен известному ряду Спицера, оценка сверху для суммы которого была недавно получена в [1].

* С.-Петербургская химико-фармацевтическая академия, кафедра высшей математики, ул. проф. Попова, 14, 197376 С.-Петербург, Россия; e-mail: l_rozovski@mail.ru

1) Исследование выполнено при поддержке программы «Ведущие научные школы» (грант НШ 638.2008.1) и РФФИ 10-01-00242-а. 